\title{
BMJ Open The Relative Effectiveness of Pumps Over MDI and Structured Education (REPOSE): study protocol for a cluster randomised controlled trial
}

\author{
David White, ${ }^{1}$ Norman Waugh, ${ }^{2}$ Jackie Elliott, ${ }^{3}$ Julia Lawton, ${ }^{4}$ Katharine Barnard, ${ }^{5}$ \\ Michael J Campbell, ${ }^{6}$ Simon Dixon, ${ }^{6}$ Simon Heller, ${ }^{3}$ on behalf of the REPOSE group
}

To cite: White D, Waugh N, Elliott J, et al. The Relative Effectiveness of Pumps Over $\mathrm{MDI}$ and Structured Education (REPOSE): study protocol for a cluster randomised controlled trial. BMJ Open 2014:4:e06204 doi:10.1136/bmjopen-2014006204

- Prepublication history for this paper is available online. To view these files please visit the journal online (http://dx.doi.org/10.1136/ bmjopen-2014-006204).

Received 25 July 2014 Revised 15 August 2014 Accepted 18 August 2014

CrossMark

For numbered affiliations see end of article.

Correspondence to Professor Simon Heller; s.heller@sheffield.ac.uk

\section{ABSTRACT}

Introduction: People with type 1 diabetes (T1DM) require insulin therapy to sustain life, and need optimal glycaemic control to prevent diabetic ketoacidosis and serious long-term complications. Insulin is generally administered using multiple daily injections but can also be delivered using an infusion pump (continuous subcutaneous insulin infusion), a more costly option with benefits for some patients. The UK National Institute for Health and Care Excellence (NICE) recommend the use of pumps for patients with the greatest need, citing insufficient evidence to approve extension to a wider population. Far fewer UK adults use pumps than in comparable countries. Previous trials of pump therapy have been small and of short duration and failed to control for training in insulin adjustment. This paper describes the protocol for a large randomised controlled trial comparing pump therapy with multiple daily injections, where both groups are provided with high-quality structured education.

Methods and analysis: A multicentre, parallel group, cluster randomised controlled trial among 280 adults with T1DM. All participants attended the week-long dose adjustment for normal eating (DAFNE) structured education course, and receive either multiple daily injections or pump therapy for 2 years. The trial incorporates a detailed mixed-methods psychosocial evaluation and cost-effectiveness analysis. The primary outcome will be the change in glycosylated haemoglobin (HbA1c) at 24 months in those participants whose baseline $\mathrm{HbA1c}$ is at or above $7.5 \%$ $(58 \mathrm{mmol} / \mathrm{mol})$. The key secondary outcome will be the proportion of participants reaching the NICE target of an $\mathrm{HbA} 1 \mathrm{C}$ of $7.5 \%$ (58 mmol/mol) or less at 24 months.

Ethics and dissemination: The protocol was approved by the Research Ethics Committee North West, Liverpool East and received Medicines and Healthcare products Regulatory Agency (MHRA) clinical trials authorisation. Each participating centre gave National Health Service R\&D approval. We shall disseminate study findings to study participants and through peer reviewed publications and conference presentations, including lay user groups.

Trial registration number: ISRCTN 61215213.

\section{Strengths and limitations of this study}

- Multicentre randomised controlled trial.

- The first randomised trial comparing insulin pumps with multiple daily injections, where both groups have received high-quality structured education.

- Long-term follow-up, with a 24-month primary outcome.

- Comprehensive psychosocial evaluation and cost-effectiveness analysis.

Blinding of participants is not possible.

\section{INTRODUCTION}

People with type 1 diabetes mellitus (T1DM; around 250000 individuals in the UK) have lost the ability to make insulin due to autoimmune destruction of the insulin secreting $\beta$ cells within pancreatic islets. Treatment with an external source of insulin is essential and life long. In the short-term insulin prevents diabetic ketoacidosis, a potentially fatal condition. In the long term, therapy is designed to keep blood glucose as close to normal as possible, to prevent microvascular complications such as retinopathy, neuropathy and nephropathy and to reduce the risk of macrovascular disease. ${ }^{1}$ A further aim of treatment is to achieve as good a quality of life (QoL) as possible, particularly since appropriate self management of the condition is challenging and arduous, demanding the implementation of complex skills.

Insulin is generally administered by intermittent subcutaneous injection with the dose adjusted according to eating, physical activity and current blood glucose levels. Traditionally, insulin was given twice a day, often as premixed insulin, but such an approach imposes a rigid lifestyle on patients and makes it difficult to maintain blood glucose levels close to 
normal. Most individuals require intensive insulin therapy to maintain tight glycaemic control. This approach and its integration within flexible lifestyles is promoted in dose adjustment for normal eating (DAFNE) ${ }^{2}$ and similar structured training courses. ${ }^{3}$ Flexible intensified insulin therapy involves the combination of quick-acting insulin before eating (altered according to the amount of carbohydrate) and long-acting background 'basal' insulin to control blood glucose in between meals. Basal insulin is generally given twice daily.

The combination is generally referred to as 'multiple daily injections', or MDI. It often involves a total of 5, even 6 injections a day, frequent checks of blood glucose from finger prick samples using a portable meter and dose adjustment based on the amount of carbohydrate eaten at each meal. Insulin given subcutaneously cannot reproduce the physiology of insulin secretion of individuals without diabetes due to the limitations of insulin prepared for therapeutic use and its delivery into the systemic rather than the portal circulation. The relatively slow rate of insulin absorption leads to postprandial hyperglycaemia and often postabsorptive hypoglycaemia, particularly at night. Short-acting and long-acting insulin analogues have more physiological profiles but cannot yet reproduce those observed in people without diabetes. ${ }^{4}$ A systematic review of clinical trials of insulin analogues involving people with T1DM reported only minor advantages compared to human insulin. ${ }^{5}$

Thus, while keeping blood glucose close to normal can delay or prevent complications, it requires considerable personal effort, and may bring with it frequent periods of hypoglycaemia. The manifestations of hypoglycaemia range from the need to ingest quick-acting carbohydrate to correct mild symptoms, to behaviour that can be socially aversive, cerebral dysfunction, loss of consciousness, through to major episodes of coma and seizure. The inability of MDI therapy to control blood glucose tightly without an attendant risk of hypoglycaemia results in many people keeping blood glucose at higher than clinically recommended levels. A significant proportion go on to develop serious diabetic complications which reduce both the length and quality of their lives. ${ }^{1}{ }^{6}$ There is therefore an urgent need for better methods of insulin delivery.

Insulin can also be administered using an infusion pump (the size of a small mobile phone), which delivers insulin continuously under the skin via a small plastic tube and cannula (Continuous subcutaneous insulin infusion, CSII).$^{7} 8$ This is not new technology, the first trials took place in the 1970's, but the devices have since improved and are now more widely used, particularly in the USA and some European countries. The devices are filled with reservoirs of quick-acting insulin, usually an insulin analogue ${ }^{9}$ and can supply the insulin needed for both background replacement and to cover meals. When infused at low rates in between eating, pumps mimic basal insulin secretion and insulin is generally delivered more consistently and accurately than is achievable by long-acting insulin injections. Rapidly infused insulin boluses, conveniently delivered from the pump at the push of a button and controlled by the individual, cover each meal.

Pump therapy is more expensive than MDI, with pumps costing around £2500-3000 each plus £1500 a year extra for running costs, mainly consumables. The marginal cost per annum over MDI was around $£ 1800$ in 2007, including both capital and revenue costs. ${ }^{10}$ The potential advantages are a more stable blood glucose, reduced risk of hypoglycaemia, enhanced QoL and a more flexible lifestyle. Pump treatment may deliver insulin more effectively than MDI but does not provide a technological 'cure'. Indeed, optimal usage still requires frequent blood glucose monitoring by the user with careful thought needing to be given to adjustment of both the background rates-particularly during the night - and the insulin dose needed at each meal.

It is estimated that only $6 \%$ of UK adults with T1DM use pumps, which is lower than in most comparable countries. ${ }^{11}$ In contrast, they may be used by as many as $40 \%$ of those with T1DM in the USA. ${ }^{12}$ Proponents of pump treatment have proposed that far more people should be offered this treatment in the UK and have suggested that current policies are depriving many of the opportunity to improve glycaemic control, reduce hypoglycaemia and improve QoL. ${ }^{13}$

There have been two appraisals of pumps by the UK's National Institute for Health and Care Excellence (NICE), both supported by technology assessment reports that reviewed the evidence on clinical and costeffectiveness. The first report ${ }^{14}$ noted that there were no trials of pumps against 'best MDI' with long-acting and short-acting analogue insulins; that some trials had unequal amounts of education in the arms (with more in the pump arms); and trials had focused on easily measurable outcomes such as glycosylated haemoglobin (HbAlc), rather than on benefits such as flexibility of lifestyle and QoL. The report recommended trials of pumps against analogue-based MDI. An updated report ${ }^{10}$ found that few such trials had been undertaken - one in children, and three in adults. The three in adults were small (a total of 103 participants) and had short follow-up. Furthermore, those participating were different to those considered suitable for pumps by NICE, which advises patients use analogue-based MDI before pump therapy. In 2008, NICE recommended extending pump treatment to those adults with T1DM experiencing problems with hypoglycaemia particularly when this limits their ability to improve glycaemic control.

\section{Why do REPOSE?}

The DAFNE course is a 1-week structured course teaching skills in insulin self-adjustment and carbohydrate counting, ${ }^{2}$ currently being delivered in 76 centres across the UK and Ireland (with over 31000 individuals having attended). In many DAFNE centres, reimbursement for 
pump use is conditional on patients having attended a DAFNE education course and, hence, some people undertake DAFNE training with the intention of moving to pump treatment thereafter. It has been our clinical experience that many such individuals decide not to switch to pump therapy after attending a DAFNE course, having realised that what they had actually required was training in insulin self-adjustment rather than a different technical way of delivering insulin. Importantly, trials and observational studies of high-quality training alone (with MDI), show benefits in blood glucose control, hypoglycaemia and QoL which are as good if not better than those reported after pump therapy. ${ }^{2} 1516$

One problem with the existing evidence base is that patients allocated to pumps may have received more training and attention than those treated using MDI. A recent observational Italian study ${ }^{17}$ of pump therapy and MDI, where both groups received intensive education, concluded that it might be the education which made most difference. To our knowledge, no randomised trials in adults have compared pump treatment with MDI where the same structured training in insulin adjustment has been given, so the added benefit of the pump technology is currently unclear. There is an urgent need to establish this, and identify the individuals who are most likely to benefit from using a pump. This will enable healthcare systems to determine the proportion of adults with T1DM that would benefit from pump therapy. A randomised controlled trial is needed to establish these outcomes without bias.

In the UK, NICE has approved the use of pumps in adults with T1DM with the greatest need, such as inability to achieve reasonable control without 'disabling hypoglycaemia'. This is in international terms, a fairly restricted usage. Evidence on the clinical and costeffectiveness of broader availability of pump therapy relative to MDI is required. Past trials may not be a good guide; they may have excluded people with persistent mild-to-moderate hypoglycaemia due to hypoglycaemic unawareness, who may be those with most to gain.

The aim of REPOSE is to assess whether pump therapy provides added benefit compared to optimised MDI therapy, after receiving high-quality structured education. We hypothesise that much of the benefit of pumps may come from the retraining and education in insulin use given to enable patients to use pumps safely. The trial builds on pilot work in which a pump course using DAFNE principles was developed. Recruitment to REPOSE is now complete and the trial is in follow-up.

\section{METHODS}

\section{Study design}

REPOSE is a multicentre, parallel group, cluster randomised controlled trial, in which 280 adults with T1DM were recruited at seven centres across the UK. Participants were allocated a place on a week-long DAFNE course. The course groups were then randomly allocated in pairs to either pump or MDI treatment. Following the course, participants receive the trial treatment for 2 years, from the secondary care centres they usually attend. Outcome measures are being collected at baseline (up to 3 weeks prior to the DAFNE course) and 6, 12 and 24 months postcourse.

A detailed psychosocial study involving both quantitative and qualitative methodology is being undertaken. A repeated measures, longitudinal questionnaire study is exploring differences in outcomes between the two trial arms and the short-term and long-term predictors and mediators of outcomes. The time-points for follow-up have been selected to capture both short-term and longterm post-treatment changes in psychosocial outcomes. A subsample of 45 participants (25 in the pump arm and 20 in the MDI arm) took part in repeat qualitative interviews (post course and at 6 months). The purpose of these interviews is to aid interpretation of the trial findings by identifying, exploring and understanding any differences and similarities in how participants manage their diabetes using a pump and MDI treatment after their courses and over time.

The study also includes a detailed economic analysis to help guide future recommendations.

\section{Setting}

Secondary care centres (three in Scotland, four in England) with experience both in delivering highquality structured education using DAFNE and delivering pump therapy in adults with T1DM. We identified two potential reserve sites in case of difficulties with recruitment, one of which has been activated. Each centre was asked to recruit 40 participants to 3 pump and 3 MDI courses (5-8 patients on each course) over 11 months. A list of participating centres can be found at the end of this paper.

\section{Participants}

Inclusion criteria: adults with T1DM for at least 12 months; aged 18 years and above; willing to undertake intensive insulin therapy with self-monitoring of blood glucose levels (SMBG), carbohydrate counting and insulin self-adjustment; who have no preference to either pump or MDI and are happy to be randomised; and, who have a need for structured education to optimise diabetes control.

Exclusion criteria: individuals who have already completed a diabetes education course; have used a pump in the past 3 years or, have strong clinical indications for pump therapy in the view of the investigator or, have a strong desire for pump therapy; renal impairment with a chance of needing renal replacement therapy within the next 2 years; uncontrolled hypertension (diastolic blood pressure $>100 \mathrm{~mm} \mathrm{Hg}$ and/or sustained systolic level $>160 \mathrm{~mm} \mathrm{Hg}$ ); a history of heart disease within the past 3 months; severe needle phobia; a current history of alcohol or drug abuse; serious or unstable conditions to preclude safe participation; recurrent skin infections; 
pregnant or planning to become pregnant within the next 2 years; taken part in any other investigational clinical trial during the 4 months prior to screening; unable to communicate in English; or, unable to give informed consent.

\section{Interventions}

\section{The standard DAFNE MDI arm}

Each course is conducted over five consecutive days, providing an average of $38 \mathrm{~h}$ of structured education and is delivered to groups of 5-8 adults aged over 18 years in an outpatient setting. Courses are delivered by diabetes specialist nurses and dietitians who have attended an educator training course, the DAFNE education programme, a seven-part programme consisting of $105 \mathrm{~h}$ of structured training.

The DAFNE curriculum uses a progressive modularbased structure to improve self-management in a variety of medical and social situations. Content is designed to deliver key learning topics at the appropriate time during the week. In this way, knowledge and skills are built up throughout the course with active participant involvement and problem solving as key methods of learning. The key modules are: what is diabetes? food and diabetes; insulin management; management of hypoglycaemia; sick day rules. Lesson plans give guidance on timing and a student activity section serves to give an idea of expected responses. Each meal and snack during the course is used as an opportunity to practise carbohydrate estimation and insulin dose adjustment.

MDI participants are using insulin analogues (a quickacting insulin analogue and twice daily injections of insulin detemir, a medium duration analogue).

\section{The DAFNE pump arm}

The 5-day structure of the standard adult DAFNE course has been maintained while modifying the course to incorporate the additional skills and learning outcomes of pump therapy. Thus, the principles of insulin dose adjustment taught on the adult course are maintained. The additional components of the course have been tested in a pilot study undertaken prior to the trial. The need to introduce 'pump skills' requires the addition of a precourse session delivered individually, run 1-2 weeks before the 'proper' DAFNE course.

Pump participants are using a Medtronic Paradigm Veo insulin pump (Model X54). Short-acting analogue insulin is being used, since in a meta-analysis ${ }^{9}$ this has been shown to lower HbAlc to a greater extent than traditional soluble insulin.

Fidelity testing of pump courses was undertaken in order to assess whether courses were delivered according to DAFNE philosophy and principles and that the educators had the necessary skills to deliver these principles. Standard DAFNE courses were not tested as there is a rigorous quality assurance programme of MDI courses in standard care.
All participants in both groups are invited to an additional DAFNE follow-up group session at 6 weeks post course, which is standard for DAFNE course attendees.

The insulin pumps include a Medtronic Bolus Wizard, to aid calculation of insulin doses. To reduce any potential bias, MDI participants are also given access to a bolus calculator (Accu-Chek Aviva Expert Bolus Advisor System).

The duration of any additional diabetes-related contact is recorded throughout the trial.

Participants receive the allocated treatment for 2 years. Treatment may be changed at the discretion of the local principal investigator if self-management of diabetes has become ineffective and is considered a risk to the individual. If the participant fails to attend the pump course they are withdrawn from pump treatment.

\section{Outcomes}

Primary outcomes

- The main primary outcome will be the change in HbAlc after 2 years in those participants whose baseline $\mathrm{HbAlc}$ was at or above $7.5 \%(58 \mathrm{mmol} / \mathrm{mol})$.

- The key secondary end point will be the proportion of participants reaching the NICE target of a HbAlc level of $7.5 \%(58 \mathrm{mmol} / \mathrm{mol})$ or less after 2 years (of all participants).

HbAlc is the accepted gold-standard measure of glycaemic control. This will provide both a measure of efficacy, and a means for modelling long-term cost-effectiveness. However, it is important to note that HbAlc may not fall in patients who enter the trial with low baseline levels of HbAlc, but who might be experiencing frequent hypoglycaemia, or wish to increase dietary freedom. Success for such individuals would be an HbAlc level which is maintained or even rises slightly with a reduction in the frequency of hypoglycaemia. We are including such patients since they can provide important information about QoL and the potential of pump therapy to reduce rates of hypoglycaemia. Since their glycaemic control may not alter, including their HbAlc data would reduce our statistical power to establish improvement in our primary end point. We are therefore powering the trial on the number of participants with a baseline HbAlc above or equal to $7.5 \%$ $(58 \mathrm{mmol} / \mathrm{mol})$ and in whom a fall would reflect worthwhile improvements in glycaemic control.

Since HbAlc can be measured using different techniques, we are ensuring standardisation by testing in a central laboratory, as well as in a local lab. We are also conducting a substudy, to determine the variation between central and local results. This will, in part, inform a decision as to the acceptability of using local lab values where central values are missing.

\section{Secondary outcomes}

\section{Biomedical outcomes}

The following outcomes are being measured at 6,12 and 24 months:

- Episodes of moderate and severe hypoglycaemia (count).

- Insulin dose (units/kg body weight). 
- Body weight $(\mathrm{kg})$.

- Lipids (high-density lipoprotein (HDL) cholesterol, total cholesterol).

- Proteinuria (albumin creatinine ratio-a sensitive measure of proteinuria).

Hypoglycaemia: We are recording episodes of both severe and moderate hypoglycaemia and specifically recording episodes at night (those occurring between 23:00 and 7:00). We are using a standard definition of severe episodes (an episode leading to cognitive impairment sufficient to cause either coma or requiring the assistance of another person to recover). ${ }^{18}{ }^{19}$ The number of severe episodes are reliably recorded by patients for up to 1 year. ${ }^{20}$ Pumps have been shown to reduce hypoglycaemia in some studies but since DAFNE and similar educational interventions are also associated with falls in severe episodes ${ }^{21} 22$ we may have insufficient power to detect a difference between the two groups.

During the last NICE appraisal of pump therapy, the question of the impact of moderate hypoglycaemia was raised. The modelling had included only severe hypoglycaemia, and the point was made that moderate hypoglycaemia, sufficient to interrupt activities of daily living, might, because of greater frequency, have more cumulative effect on QoL than severe hypoglycaemia. We are therefore also recording rates of moderate hypoglycaemia in an attempt to increase power and identify the ability of pumps to reduce rates of hypoglycaemia. We define moderate hypoglycaemia as 'any episodes which could be treated by that individual, but where hypoglycaemia caused significant interruption of current activity, such as having caused impaired performance or embarrassment or having been woken during nocturnal sleep'. Since these episodes are more frequent, reliable recall of such events is unlikely to be sustained for more than a few weeks. We therefore ask participants to record the number and timing of moderate episodes over the 4 weeks prior to each follow-up visit. We used this approach successfully to record the frequency of mild episodes in a recent epidemiological study of hypoglycaemic burden in diabetes. ${ }^{18}$

We will assess the impact of both, by comparing QoL in those with only moderate hypoglycaemia, versus those with moderate and severe.

Insulin dose and body weight: pump treatment may result in the use of less insulin leading to a favourable effect on body weight. We record total insulin dose at each time point and calculate units/ $\mathrm{kg}$ body weight.

Lipids and proteinuria (as measured by albumin creatinine ratio): A recent study ${ }^{23}$ reported little difference in HbA1c on pump therapy compared to MDI but found less progression to microalbuminuria in the pump group, and also lower cholesterol levels.

\section{Safety outcomes}

Diabetic ketoacidosis is being recorded through the assessment of serious adverse events at any point in time during the trial.
Quantitative psychosocial outcomes

The following are being measured using a participant self-completed questionnaire administered at 6,12 and 24 months:

- QoL (both generic and diabetes specific)

- Fear of hypoglycaemia

- Treatment satisfaction

- Emotional well-being

There has been limited examination of the impact of pump therapy on these areas, on how and why these may change over time and why individuals are able or unable to use pump therapy to improve glycaemic control. Rubin and Peyrot ${ }^{24}$ reviewed the evidence on 'patient reported outcomes' and concluded that at present, there is little evidence that pump therapy improved them.

QoL: Diabetes-specific QoL is being assessed using the scale (DSQOLS), a reliable and valid measure. ${ }^{25}$ Specifically designed for the German study on which UK DAFNE is based, it is included to facilitate important comparisons between the UK and German studies. In addition, generic measures of QoL, the WHO QoL Abbreviated Questionnaire (WHOQOLBREF) ${ }^{26}$ and functional health status using the Short Form Health Survey $(\mathrm{SF}-12)^{27}$ and EuroQoL (EQ5D) ${ }^{28}$ are being used. The SF-12 will facilitate comparison with 'healthy controls' and other long-term conditions. The SF-12 and EQ5D will also be used by the health economists to derive health economic data.

Fear of hypoglycaemia: The Hypoglycaemia Fear Survey ${ }^{29}$ is a well validated psychometric tool assessing participants' fear of hypoglycaemia both overall and separately for behaviour and worry. A specific benefit to the survey is that it may be able to identify participants who are likely to maintain high-blood glucose levels, thus aiding understanding of potential reasons for poor glycaemic control. A study by Nixon and Pickup ${ }^{30}$ in people who had been using a pump for an average of 5 years, found that fear of hypos remained a problem.

Treatment satisfaction: The Diabetes Treatment Satisfaction Questionnaire ${ }^{31}$ has proven to be highly sensitive in clinical trials. ${ }^{32}$ Treatment satisfaction refers to an individual's subjective appraisal of their experience of treatment, including ease of use, side effects and efficacy. Improvements in satisfaction are not necessarily accompanied by improvements in QoL; treatment satisfaction can be high despite diabetes having a negative impact on QoL, which is why it is important to measure both separately.

Emotional well-being: The Hospital Anxiety and Depression Scale ${ }^{33}$ measures anxiety on one subscale and depression on another through the use of seven questions for each characteristic. It is important to measure emotional well-being in the trial as participants may find it easier to manage their condition after DAFNE education or with one of the treatments. This might have a substantial effect on their emotional wellbeing that the QoL measures are not sensitive enough to pick up. 


\section{Qualitative substudy}

Participant interviews conducted within 2 weeks of course completion explored:

- Experiences of diabetes management prior to the trial.

- Understandings of the trial, experiences of recruitment and motivation for participation.

- Reactions to, and views about, randomisation outcome.

- Expectations and concerns about trial participation and (if relevant) change to pump therapy.

- Experience of and views about their course and (if relevant) change to pump therapy.

- Likes/dislikes of pump or MDI treatment.

- Changes made to diabetes management since the course and any short-term/long-term goals set.

Follow-up interviews at 6 months were used to explore:

- Continuities and changes in how participants managed their diabetes since their last interview and the reasons for these.

- Barriers and facilitators to sustaining intensive insulin therapy using a pump or MDI treatment.

- Reasons for pump discontinuation and/or treatment non-adherence.

- Any changes in participants' perceptions and attitudes towards their disease and the reasons for these changes.

In order to contextualise and interpret participants' responses, additional issues and areas were also explored in their baseline and 6-month interviews. These included: participants' work and family commitments; support received from family and friends; their contact with diabetes health professionals within and without the trial; their views about the information, support and clinical care received; and, any unmet needs for information and support.

\section{Health economics}

We will undertake a health economic evaluation to address the question 'What is the cost effectiveness of pump therapy compared to MDI in patients receiving the DAFNE structured education programme?' We will undertake both a within trial and a modelled patient lifetime analysis, the latter being the primary focus of the evaluation. The following outcomes will be measured.

- Quality adjusted life years (QALYs) for intervention and comparator modelled over a lifetime horizon.

- Total costs of intervention and comparator over a lifetime horizon, accounting for the costs of complications and adverse events as well as initial intervention costs.

- QALYs observed over 2-year trial period in each trial arm.

- Cost of each trial arm over 2 years

- Incremental cost-effectiveness ratios (ICERs) over a lifetime horizon and over the 2-year trial period

- Uncertainty surrounding the ICERs (cost-effectiveness plane, cost-effectiveness acceptability curve and frontier, probability of cost-effectiveness at willingness-to-pay thresholds of $£ 20000$ and $£ 30000$ per QALY, deterministic sensitivity analyses).

The within-trial analysis will be conducted in line with Ramsey et $a l \mathrm{~s}^{34}$ recommendations for cost-effectiveness analysis alongside clinical trials. Specifically, the analysis will use unit costs consistent with measured resource use, use EQ-5D as the measure of health outcome, and follow the guiding principles outlined for the analysis of economic measures. The baseline analysis will be based on complete cases, with an additional sensitivity analysis using multiple imputation to account for missing data using the ICER procedure within STATA. Patient-level data is being collected for equipment, drugs and NHS contacts. Unit costs will be taken from standard sources (NHS Reference Costs, British National Formulary, PSSRU), plus a survey of pump costs across the recruiting centres. Utility scores for each patient will be calculated using the UK EQ-5D. QALYs for each patient will be estimated by calculating the area under the curve defined by EQ-5D utility score, mortality and length of follow-up.

The long-term modelling analysis will use the Sheffield Type 1 Diabetes Policy model-which has been developed as part of the national DAFNE Research Programme-in combination with data from the trial. ${ }^{35}$ It will be conducted in line with the NICE reference case for economic evaluations (NIHR, 2008) and the Guidelines for computer modelling of diabetes and its complications. ${ }^{36}$ The model covers the entire T1DM care pathway including initiation, management, outcomes and long-term complications. The analysis will include overall cost-effectiveness, analysis of subgroups and use methods of probabilistic sensitivity analysis to quantify remaining uncertainties in costs and benefits.

\section{Sample size}

It is generally accepted that a difference of $0.5 \%$ $(5.5 \mathrm{mmol} / \mathrm{mol})$ in HbA1c is clinically worthwhile. To detect this difference with a SD of $1 \%$ at $80 \%$ power and $5 \%$ two-sided significance using a $\mathrm{t}$ test requires 64 patients per group for participants over $7.5 \% \mathrm{HbA1c}$. To allow for a clustering effect of the educators, with an average of seven patients per DAFNE group and a within-course intraclass correlation coefficient (ICC) of 0.05 , which is common in diabetes care, the sample size increases to 84 . Allowing also for a $10 \%$ drop out, the sample size per group becomes 93. Audit of the DAFNE database shows that $75 \%$ of participants have an HbAlc of over $7.5 \%$, so overall we require 124 participants per group, that is, 248 in total. We planned to recruit 280 participants which increases the power to $85 \%$ but which allows for some variation in drop-out rates and the proportion of patients with HbAlc $>7.5 \%$. However, our final recruitment was 321.

\section{Assignment to control and intervention}

Informed consent was obtained by the principal investigator or educator, trained in good clinical practice. After consent, participants were allocated to a REPOSE 
DAFNE course, depending on the participants' availability. Courses were randomised in pairs, stratified by centre, to either DAFNE + pump or DAFNE + MDI, by a statistician using a computerised system within Sheffield Clinical Trials Research Unit (CTRU). The final pair of courses at each centre were randomised using minimisation, based on the participants' most recent available HbAlc result (HbA1c >or equal to $7.5 \%$ vs $<7.5 \%$ ).

\section{Data collection and management}

A case report form (CRF) is completed by the educator at each study visit, with the participant present. A psychosocial questionnaire pack is self-completed by the participant prior to the visit in order to reduce potential reporting bias. CRF data is entered at local centres by educators/administrators onto a centralised online database managed by Sheffield CTRU. Participant completed questionnaires are posted to Sheffield CTRU and entered centrally by Sheffield CTRU administrators. Data is stored in accordance with Sheffield CTRU standard operating procedures.

Data quality is being checked using a combination of point of entry validation, $10 \%$ verification of data entry, postentry validation and other ad hoc validation by the trial monitoring committees and CTRU statisticians. A copy of the data management plan will be provided on request.

\section{Statistical analysis}

The primary analysis will be a linear model of HbAlc at 2 years with treatment (pump/MDI), centre and baseline $\mathrm{HbAlc}$ as fixed effect covariates and course as a random intercept. The analysis will be undertaken using generalised estimating equations (GEE). We plan to use Poisson regression on the number of moderate or severe hypoglycaemic episodes in 2 years with the same covariates, again using a GEE approach to account for clustering; however, we will test the adequacy of Poisson regression against alternatives such as the negativebinomial and zero-inflated Poisson regressions. We will use an intention-to-treat analysis, but will explore the effect of switching by conducting a per protocol secondary analysis. Participants who dropped out before receiving the intervention were substituted where possible, to ensure the courses were run with adequate numbers of participants, but these individuals will not form part of the analysis set. A copy of the statistical analysis plan will be provided on request.

\section{Monitoring}

Three committees were established to govern the conduct of this study: an independent Trial Steering Committee (TSC) and Data Monitoring and Ethics Committee (DMEC) and day to day running by an internal Trial Management Group (TMG). The composition of the TSC and DMEC are listed at the end of this paper. These committees function in accordance with Sheffield CTRU standard operating procedures.
Emerging evidence is being monitored through;

- 6-monthly updating searches.

- Auto-alerts set up in Medline.

- General surveillance by project team, for example at diabetes conferences.

- Feedback from members of the TSC.

The study is classed as a low-risk clinical trial of an investigational medicinal product (CTIMP) by the Medicines and Healthcare Regulatory Authority (MHRA) and we adhere to the reporting requirements considered appropriate for such trials. Details of any adverse events are collected at each follow-up time point. Participants are also encouraged to contact their local diabetes team if they experience any adverse health events. Serious adverse events are assessed by the local principal investigator and reported to Sheffield CTRU within $24 \mathrm{~h}$, in accordance with Sheffield CTRU standard operating procedures. Adverse events are reviewed by the three study oversight committees.

On site monitoring of sites is being undertaken before, during and after the trial, with a minimum of three visits at each site, undertaken by the trial monitor.

\section{Ethical considerations \\ Risks}

Intensive insulin regimens are commonly used in this age group, and it is difficult to envisage significant additional risks from participation in this study. Early experience using pump therapy documented increased risk of diabetic ketoacidosis but this has not been substantiated in more recent trials and should be largely preventable by appropriate sick day rules training, a standard part of the DAFNE curriculum. There is a small risk of cannula site infection with insulin infusion but this is minimised by proper instruction in site care and regular change of site. Some forms of intensified insulin therapy are reported to increase severe hypoglycaemia risk but in the parent programme for DAFNE and more recently in a clinical DAFNE audit, we have shown a reduction in severe hypoglycaemia rate and diabetic ketoacidosis at 1 year after DAFNE training. ${ }^{21} 22$

\section{Anticipated benefits}

Both courses aimed to provide adults with the skills to closely match their insulin dose to their food choice and regularly correct their blood sugar. The anticipated benefits are therefore improved blood glucose control, QoL and self-efficacy.

\section{Patient recruitment}

All participants received patient information literature describing the anticipated risks and benefits of taking part and had the opportunity to ask questions prior to giving informed consent.

\section{Other ethical issues regarding study participation}

All blood samples and biomedical measurements are routinely taken in clinic. Blood glucose is measured at 
home by a meter from blood taken by finger prick sampling (SMBG), which people with diabetes perform several times each day.

No new drugs or insulins are being used. All are licensed and currently in use in the UK with this age group. Intensive insulin regimens are currently being used in many UK centres, with some starting this regimen from the time of diagnosis.

Individuals who participate are fully aware that they are not guaranteed the use of the pump either during or at the end of the study. However, those pump participants who express a preference to continue therapy and have measurable evidence of benefit (HbAlc reduction greater than $0.5 \%$; reduction in hypoglycaemia rate; documentable improvement in a QoL outcome) will be supported by their diabetes team in seeking local NHS approval for continuation of pump therapy. Participants reverting to MDI will be reassessed at 4-6 months; if their QoL or HbAlc or hypoglycaemia status has deteriorated on MDI, a direct approach to the local commissioners or health board for conversion to pump therapy will be made.

Participants are free to withdraw from the study at any time. Those who wish to switch back to MDI are seen by one of the centre DAFNE educators who work with them to ensure they have the necessary information to use their DAFNE skills with multiple injections.

\section{Service users}

As part of the DAFNE NIHR programme grant, 15 DAFNE graduates were recruited to act as a 'user group' and contribute to different aspects of the work. The group agreed to serve on steering groups providing opinion and assistance in refining research projects. We invited two members to join both the steering group and other investigator meetings. One of the project group is a pump user.

\section{Dissemination}

We shall disseminate findings to study participants and through peer reviewed publications and conference presentations, including presentations to lay user groups. Trial updates will be provided on the study website.

\section{DISCUSSION}

\section{Progress so far (June 2014)}

Recruitment is now closed at all participating centres and follow-up is ongoing. A total of 321 participants were recruited and 46 DAFNE courses administered, more than the original target of 280 participants and 42 DAFNE courses. The targets were increased to counter a higher than expected number of withdrawals prior to the DAFNE courses. We had anticipated the potential for recruitment problems and so had recruited a reserve centre which was introduced to facilitate a pair of courses, while an existing centre recruited to a seventh and eighth course. We plan to complete follow-up in July 2015 and report results in early 2016.

Author affiliations

${ }^{1}$ Clinical Trials Research Unit, University of Sheffield, Sheffield, UK

${ }^{2}$ Division of Health Sciences, Warwick Medical School, University of Warwick, Warwick, UK

${ }^{3}$ Academic Unit of Diabetes, Endocrinology and Metabolism, University of Sheffield, Sheffield, UK

${ }^{4}$ Centre for Population Health Sciences, University of Edinburgh, Edinburgh, UK

${ }^{5}$ Human Development and Health, Faculty of Medicine, University of Southampton, Southampton, UK

${ }^{6}$ School of Health and Related Research, University of Sheffield, Sheffield, UK

Acknowledgements The authors would like to thank the patients who are taking part in this study. The research is sponsored by Sheffield Teaching Hospitals NHS Foundation Trust. The clinical sites taking part are: Cambridge University Hospitals NHS Foundation Trust, NHS Dumfries and Galloway, NHS Greater Glasgow and Clyde, Harrogate and District NHS Foundation Trust, King's College Hospital NHS Foundation Trust, NHS Lothian, Nottingham University Hospitals NHS Trust and Sheffield Teaching Hospitals NHS Foundation Trust. The authors would like to thank Jane Baillie, Helen Brown, Karen Callaby, Katy Davenport, Sarah Donald, Leila Faghahati, Sara Hartnell, Allison Housden, Kulbir Kaur Pabla, Nicola Croxon, Sheena Macdonald, Muna Mohammed, Vicky Steel, Katy Valentine, Pamela Young, Ann Boal, Patsy Clerkin, Lynn Doran, Joanne Flynn, Emma Gibb, Hilary Peddie, Bernie Quinn, Janice Shephard, Janet Carling, Ann Collins, Laura Dinning, Christine Hare, Joyce Lodge, Sutapa Ray, Debora Brown, Jenny Farmer, Alison Cox, Chris Cheyette, Pratik Choudhary, Linda East, June Ellul, Katherine Hunt, Kimberley Shaw, Ben Stothard, Lucy Diamond, Lindsay Aniello, Debbie Anderson, Kathy Cockerell, Vida Heaney, Alison Hutchison, Nicola Zammitt, Gayna Babington, Gail Bird, Janet Evans, Tasso Gazis, Nicola Maude, Karen Nunnick, Dawn Spick, Laura Fenn, Carla Gianfrancesco, Valerie Gordon, Linda Greaves, Susan Hudson, Valerie Naylor, Chloe Nisbet and Karen Towse for contributing to participant recruitment, data collection and intervention delivery; Jackie Kirkham and David Rankin for contributing to the qualitative research; Lucy Carr for central trial administration; Pamela Royle for literature searches to monitor emerging evidence; Sharon Walker for central DAFNE assistance; and, Pauline Cowling for fidelity assessment. The independent trial steering committee members are: Richard Holt (chair), Sean Dinneen, Dawn Kitchener, Jane Morgan, Mark Mullee, John Pickup, Brian Trench. The independent data monitoring and ethics committee members are: Robert Tattersall (chair), Paul Ewings, Florence Finlay White. The following collaborators have made a contribution to the paper: Stephanie Amiel, Anita Beckwith, Cindy Cooper, Mark Evans, Fiona Green, Peter Hammond, Alan Jaap, Brian Kennon, Robert Lindsay, Peter Mansell, Helen Rogers, W Henry Smithson, Carolin Taylor, Gill Thompson, Candice Ward and Emma Whatley contributed to the design of the protocol; Munya Dimairo, Mike Bradburn and Ellen Lee contributed to the statistical analysis plan; Hasan Basarir, Alan Brennan, Jen Kruger and Praveen Thokala contributed to the health economic analysis plan; Timothy Chater and Kirsty Pemberton contributed to the data management plan; Diana Papaioannou and Gemma Hackney contributed to the monitoring plan.

Contributors DW, NW, JE, JL, KB, MJC, SD and SH contributed to the design of the protocol and drafted or critically revised the manuscript. All authors read and approved the final manuscript.

Funding This research is funded by the UK Health Technology Assessment Programme (project number 08/107/01), who monitor progress and quality through bi-annual reports. The full report will be peer reviewed and published in Health Technology Assessment. The NIHR encourages grant holders to seek external peer reviewed publication. We also acknowledge the financial support of the Research and Development Programmes of the Department of Health for England and the Scottish Health and Social Care Directorates, and of Medtronic Ltd., who provided the insulin pumps for the trial. Medtronic Ltd. had no involvement in the design of the protocol or in the decision to submit this paper for publication. 
Competing interests KB sits on the global advisory board for Roche Diagnostics, Roche European Insulin Delivery Systems Advisory Board, Lifescan CHOICE Advisory Board and has received honoraria for presentations and expert advice from Roche, Janssen, Animas, Lifescan, Sanofi, AbbVie and Novo Nordisk. SH has undertaken consultancy for NovoNordisk, Eli Lilly and Sanofi Aventis, and has received speaker fees from NovoNordisk and Eli Lilly.

Ethics approval The protocol was approved by the Research Ethics Committee North West, Liverpool East. Each participating centre gave NHS Research and Development approval. The protocol received MHRA clinical trials authorisation.

Provenance and peer review Not commissioned; peer reviewed for ethical and funding approval prior to submission.

Open Access This is an Open Access article distributed in accordance with the Creative Commons Attribution Non Commercial (CC BY-NC 4.0) license, which permits others to distribute, remix, adapt, build upon this work noncommercially, and license their derivative works on different terms, provided the original work is properly cited and the use is non-commercial. See: http:// creativecommons.org/licenses/by-nc/4.0/

\section{REFERENCES}

1. The Diabetes Control and Complications Trial Research Group. The effect of intensive treatment of diabetes on the development and progression of long-term complications in insulin-dependent diabetes mellitus. N Engl J Med 1993;329:977-86.

2. DAFNE Study Group. Training in flexible, intensive insulin management to enable dietary freedom in people with type 1 diabetes: dose adjustment for normal eating (DAFNE) randomised controlled trial. BMJ (Clinical research ed) 2002;325:746.

3. Muhlhauser I, Bruckner I, Berger M, et al. Evaluation of an intensified insulin treatment and teaching programme as routine management of type 1 (insulin-dependent) diabetes. The Bucharest-Dusseldorf Study. Diabetologia 1987;30:681-90.

4. Heller SR. Insulin analogues. Curr Med Res Opin 2002;18(s1):s40-7.

5. Siebenhofer A, Plank J, Berghold A, et al. Short acting insulin analogues versus regular human insulin in patients with diabetes mellitus. Cochrane Database Syst Rev 2004(2):Cd003287.

6. Nathan DM, Cleary PA, Backlund JY, et al. Intensive diabetes treatment and cardiovascular disease in patients with type 1 diabetes. N Engl J Med 2005;353:2643-53.

7. Tamborlane WV, Bonfig W, Boland E. Recent advances in treatment of youth with type 1 diabetes: better care through technology. Diabet Med 2001:18:864-70.

8. Pickup J, Keen $\mathrm{H}$. Continuous subcutaneous insulin infusion at 25 years: evidence base for the expanding use of insulin pump therapy in type 1 diabetes. Diabetes Care 2002;25:593-8.

9. Colquitt J, Royle P, Waugh N. Are analogue insulins better than soluble in continuous subcutaneous insulin infusion? Results of a meta-analysis Diabet Med 2003;20:863-66.

10. Cummins E, Royle P, Snaith A, et al. Clinical effectiveness and cost-effectiveness of continuous subcutaneous insulin infusion for diabetes: systematic review and economic evaluation. Health Technol Assess 2010;14:iii-iv, xi-xvi, 1-181.

11. Diabetes UK. The United Kingdon insulin pump audit, 2013. http:// www.diabetes.org.uk/Documents/News/The_United_Kingdom Insulin_Pump_Audit_May_2013.pdf (accessed 14 Jul 2014)

12. Pickup J. Insulin pumps. Int J Clin Pract Suppl 2011;65:16-19.

13. Pickup JC. Are insulin pumps underutilized in type 1 diabetes? Yes. Diabetes Care 2006;29:1449-52.

14. Colquitt JL, Green C, Sidhu MK, et al. Clinical and costeffectiveness of continuous subcutaneous insulin infusion for diabetes 2004;8:iii, 1-171.

15. Muhlhauser I, Jorgens V, Berger M, et al. Bicentric evaluation of a teaching and treatment programme for type 1 (insulin-dependent) diabetic patients: improvement of metabolic control and other measures of diabetes care for up to 22 months. Diabetologia 1983;25:470-6.

16. Samann A, Muhlhauser I, Bender R, et al. Glycaemic control and severe hypoglycaemia following training in flexible, intensive insulin therapy to enable dietary freedom in people with type 1 diabetes: a prospective implementation study. Diabetologia 2005;48:1965-70.

17. Leotta SA, Altomare M, Carletti S, et al. Intensive diabetes management in type 1 diabetic patients in poor glycemic control treated with insulin pump therapy or multiple daily injections: two years follow-up. 4th International Conference on Advanced Technologies and Treatments for Diabetes, London, 2011.

18. UK Hypoglycaemia Study Group. Risk of hypoglycaemia in types 1 and 2 diabetes: effects of treatment modalities and their duration. Diabetologia 2007;50:1140-7.

19. Seaquist ER, Anderson J, Childs B, et al. Hypoglycemia and diabetes: a report of a workgroup of the American Diabetes Association and the Endocrine Society. Diabetes Care 2013;36:1384-95.

20. Pedersen-Bjergaard U, Pramming S, Thorsteinsson B. Recall of severe hypoglycaemia and self-estimated state of awareness in type 1 diabetes. Diabetes Metab Res Rev 2003;19:232-40.

21. Hopkins D, Lawrence I, Mansell P, et al. Improved biomedical and psychological outcomes 1 year after structured education in flexible insulin therapy for people with type 1 diabetes the UK DAFNE experience. Diabetes Care 2012;35:1638-42.

22. Elliott J, Jacques RM, Kruger J, et al. Substantial reductions in the number of diabetic ketoacidosis and severe hypoglycaemia episodes requiring emergency treatment lead to reduced costs after structured education in adults with type 1 diabetes. Diabet Med 2014;31:847-53.

23. Lepore $\mathrm{G}$, Bruttomesso D, Bonomo $\mathrm{M}$, et al. Continuous subcutaneous insulin infusion is more effective than multiple daily insulin injections in preventing albumin excretion rate increase in type 1 diabetic patients. Diabet Med 2009;26:602-08.

24. Rubin RR, Peyrot M. Patient-reported outcomes and diabetes technology: a systematic review of the literature. Pediatr Endocrinol Rev 2010;7(Suppl 3):405-12.

25. Bott $\mathrm{U}$, Muhlhauser I, Overmann $\mathrm{H}$, et al. Validation of a diabetes-specific quality-of-life scale for patients with type 1 diabetes. Diabetes Care 1998;21:757-69.

26. The WHOQOL Group. Development of the World Health Organization WHOQOL-BREF quality of life assessment. Psychol Med 1998;28:551-8.

27. Ware J Jr, Kosinski M, Keller SD. A 12-Item Short-Form Health Survey: construction of scales and preliminary tests of reliability and validity. Med Care 1996;34:220-33.

28. EuroQol. A new facility for the measurement of health-related quality of life. Health Policy 1990;16:199-208.

29. Cox DJ, Irvine A, Gonder-Frederick L, et al. Fear of hypoglycemia: quantification, validation, and utilization. Diabetes Care 1987;10:617-21.

30. Nixon R, Pickup JC. Fear of hypoglycemia in type 1 diabetes managed by continuous subcutaneous insulin infusion: is it associated with poor glycemic control? Diabetes Technol Ther 2011;13:93-8.

31. Lewis KS, Bradley C, Knight G, et al. A measure of treatment satisfaction designed specifically for people with insulin-dependent diabetes. Diabet Med 1988;5:235-42.

32. Secnik Boye K, Matza L, Oglesby A, et al. Patient-reported outcomes in a trial of exenatide and insulin glargine for the treatment of type 2 diabetes. Health Qual Life Outcomes 2006;4:1-8.

33. Zigmond AS, Snaith RP. The hospital anxiety and depression scale. Acta Psychiatr Scand 1983;67:361-70.

34. Ramsey S, Willke R, Briggs A, et al. Good research practices for cost-effectiveness analysis alongside clinical trials: the ISPOR RCT-CEA Task Force report. Value in Health 2005;8:521-33.

35. Thokala P, Kruger J, Brennan A, et al. Assessing the cost-effectiveness of type 1 diabetes interventions: the Sheffield type 1 diabetes policy model. Diabet Med 2014;31:477-86.

36. American Diabetes Association Consensus Panel. Guidelines for computer modeling of diabetes and its complications. Diabetes Care 2004;27:2262-65. 